\title{
Métodos de gradiente para otimização simultânea: estudo de casos de sistemas alimentares
}

\section{Gradients methods for simultaneous optimizations: case studies for food systems}

\author{
Renato Lajarim Carneiro ${ }^{1 *}$; Rui Sérgio dos Santos Ferreira da Silva²; \\ Dionísio Borsato ${ }^{3}$; Evandro Bona ${ }^{3}$
}

\section{Resumo}

Este trabalho teve como objetivo desenvolver um aplicativo com métodos de gradiente eficazes na otimização de sistemas alimentares com respostas múltiplas utilizando as funções de Derringer \& Suich, incluindo um polinômio de $4^{\circ}$ grau para a remoção das descontinuidades dessas funções. $O$ aplicativo foi testado em três sistemas alimentares selecionados na literatura especializada: 1) inativação da lipoxigenase e lípase preservando-se a atividade da fitase durante o processamento hidrotérmico da cevada; 2) otimização simultânea das respostas na formulação de misturas de proteínas; 3) otimização simultânea dos parâmetros de torração de gérmen de milho visando aplicação como ingrediente em alimentos. O programa desenvolvido mostrou ser eficiente e confiável para a otimização em sistemas alimentares multirresposta.

Palavras-chave: Otimização multirresposta, método gradiente, funções de Derringer \& Suich

\begin{abstract}
The objective of this work was to develop a software with efficient gradient methods for foods systems multiresponse optimization using the Derringer \& Suich function, including a $4^{\text {th }}$ order polynomial equation to remove non-differentiable points in that function. The software was tested in three food systems selected in specialized literature: 1) inactivated lipoxygenase and lipase and preserve phytase activity in barley during soaking; 2) simultaneous optimization of response in protein mixture formulation; 3) simultaneous optimization of parameters used in roasting process of corn germ to be used as an ingredient in foods. The program that was developed has show itself to be efficient and trustworthy for the optimization of multiresponse.
\end{abstract}

Key words: Multiresponse optimization, gradient method, Derringer \& Suich's functions

1 Bolsista de Iniciação Científica UEL. renatolajarim@pop.com.br.

2 Professor Titular do Departamento de Tecnologia de Alimentos e Medicamentos da Universidade Estadual de Londrina.

3 Professor do Departamento de Química da Universidade Estadual de Londrina.

* Autor para correspondência. 


\section{Introdução}

O desenvolvimento de novos produtos, além de considerações técnicas, legais e mercadológicas, requer também processos de otimização que possam buscar melhores condições de processamento e formulação, com alta qualidade e baixo custo (CASTRO et al., 2003).

A metodologia de superfície de resposta (MSR) é, atualmente, o mais popular conjunto de técnicas para otimização. A primeira etapa desta técnica consiste na escolha de um delineamento experimental seguida de modelagem matemática, que é realizada ajustando-se modelos lineares ou quadráticos a resultados experimentais, obtidos através de planejamentos fatoriais com ou sem ampliação (BARROS NETO; SCARMINIO; BRUNS, 2001). Após esta etapa é possível deslocar-se sobre a superfície de resposta ajustada, a fim de localizar regiões que satisfaçam condições de interesse.

Um grande problema na área de alimentos é a multiplicidade de respostas. Assim, no desenvolvimento de formulações alimentares, por exemplo, o custo dos ingredientes, bem como, as propriedades funcionais, sensoriais e nutricionais do produto requer tratamento conjunto.

Uma abordagem eficiente desse problema exige a sistematização de técnicas computacionais que possam estimar a melhor combinação dos fatores que resulte na melhor combinação possível das respostas, considerando a importância relativa de cada resposta para o sistema estudado. O emprego da função de desejabilidade (FD) é uma das técnicas que permite uma resolução para esse tipo de problema, que foi proposta inicialmente por Harrington e, posteriormente modificada por Derringer e Suich (1980). Esta metodologia já foi empregada com sucesso em sistemas alimentares que apresentavam diversas respostas (SILVA; BORSATO; SILVA, 2000; GUILLOU; FLOROS, 1993).

Além da metodologia de superfície de resposta, tem-se os métodos seqüenciais onde as etapas da otimização são praticamente as mesmas independente do método escolhido e podem ser facilmente automatizadas. Dentre os métodos seqüenciais estão os de busca direta, que se baseiam na repetição de figuras geométricas, e métodos de busca indireta, que necessitam da avaliação da primeira derivada para localizar a direção ótima do movimento.

Os métodos de gradiente, que estão entre os de busca indireta, são mais eficientes que os de busca direta e podem facilmente manipular restrições suplementares (incluindo limitações não-lineares). Porém, o emprego do gradiente era impossibilitado devido a possíveis descontinuidades na FD. Del Castillo, Montgomery e McCarville (1996) propuseram uma solução para que as descontinuidades da função de desejabilidade fossem corrigidas através de aproximações polinomiais locais de quarta ordem.

O objetivo desse trabalho foi desenvolver um aplicativo para microinformática incorporando técnicas de otimização por gradiente, adotando também um critério de parada ao final da convergência.

\section{Metodologia}

\section{Metodologia da Superficie de Resposta}

\section{Seja uma função $f\left(x_{n}\right)$ :}

- f é uma função de aproximação que representa a resposta procurada y. É uma variável dependente;

- x é uma variável controlada no delineamento experimental. É uma variável independente;

- n é o número de variáveis independentes;

As respostas $\mathrm{y}_{\mathrm{m}}$, onde $\mathrm{m}=1, \ldots, \mathrm{p}$, sendo $\mathrm{p}$ o número de respostas, podem ser escritas na forma de polinômios de segunda ordem: 
$\mathrm{y}_{\mathrm{m}}=\beta_{0, \mathrm{~m}}+\sum_{i=1}^{n} \beta_{\mathrm{i}, \mathrm{m}} \mathrm{x}_{\mathrm{i}}+\sum_{i=1}^{n} \beta_{\mathrm{ii}, \mathrm{m}} \mathrm{x}_{\mathrm{i}}^{2}+\sum_{i=1}^{n-1} \sum_{j=i+1}^{n} \beta_{\mathrm{ij}, \mathrm{m}} \mathrm{x}_{\mathrm{i}} \mathrm{x}_{\mathrm{j}} \quad$ onde:

$\mathrm{k}=$ número da iteração

onde, os parâmetros $\beta$ são coeficientes ajustáveis para cada resposta (BOX; DRAPER, 1987)

\section{Delineamento experimental}

Os delineamentos experimentais empregados freqüentemente na literatura especializada, para a superfície de resposta, são os centrais compostos com cinco níveis e os incompletos com três níveis de variação (BOX; DRAPER, 1987)

\section{Ajuste do modelo}

Para a otimização através do programa OTGRAD foram usados os coeficientes fornecidos pelos autores. Todos os modelos foram testados e confirmados pelo programa de análise estatística, sendo que todos os modelos apresentaram altos $\mathrm{R}^{2} \mathrm{e}$ foram significativos ao nível de $5 \%(\mathrm{p} \leq 0,05)$, indicando que os modelos possuem boa concordância com os valores experimentais sendo, assim, adequados para o estudo da otimização por modelagem de superfície de respostas (MSR).

\section{Método de otimização gradiente}

O método Gradiente é um modelo de otimização de busca indireta e faz o uso de derivadas para determinar a direção da busca do ponto ótimo. A otimização consiste na derivada parcial da função objetivo (FD) em relação às variáveis independentes em um determinado ponto inicial, dando origem à uma matriz gradiente unidimensional. Através dessa matriz é calculada a direção do ponto ótimo. A próxima etapa consiste em encontrar um novo ponto que satisfaça mais a função objetivo (FD) que o ponto anterior.

Esse novo ponto é dado como:

$$
\mathrm{X}^{\mathrm{k}+1}=\mathrm{X}^{\mathrm{k}}+\lambda^{\mathrm{k}} \mathrm{S}^{\mathrm{k}}
$$

$\mathrm{X}^{0}=$ ponto inicial;

$\mathrm{S}^{\mathrm{k}}=$ direção da busca;

$\lambda^{\mathrm{k}}=$ escalar que determina o tamanho do passo na direção do ponto ótimo;

À partir deste novo ponto, é feito novamente o cálculo da matriz gradiente e assim recomeça a procura por um ponto que satisfaça melhor a FD.

Foram usados três métodos de gradiente que diferem entre si no cálculo da direção da busca, $S^{k}$ (EDGAR; HIMMELBLAU, 1989). O escalar $\lambda$ ótimo é encontrado variando um valor inicial numa razão de $1 / 5$. No primeiro, o Método Gradiente comum, a direção da busca é simplesmente o oposto do próprio gradiente da FD:

$$
\mathrm{S}^{\mathrm{k}}=-\nabla \mathrm{f}\left(\mathrm{X}^{\mathrm{k}}\right)
$$

neste caso temos uma direção negativa do gradiente ("steepest descent"), onde a busca é uma minimização. Quando o objetivo for uma maximização, usa-se o oposto da FD:

$$
\mathrm{f}\left(\mathrm{x}_{\mathrm{n}}\right)_{\text {maximização }}=-\mathrm{f}\left(\mathrm{x}_{\mathrm{n}}\right)
$$

O segundo método chamado Gradiente Conjugado, desenvolvido por Fletcher e Reeves, a direção de busca é uma combinação linear da direção anterior e do gradiente da FD:

$$
S^{0}=-\nabla f\left(x^{0}\right)
$$

calcula-se a seguir um próximo ponto:

$$
X^{1}=X^{0}+\lambda^{0} S^{0}
$$

a partir de então a direção de busca S será dada como: 


$$
S^{k+1}=-\nabla f\left(x^{k+1}\right)+S^{k} \frac{\nabla^{T} f\left(x^{k+1}\right) \nabla f\left(x^{k+1}\right)}{\nabla^{T} f\left(x^{k}\right) \nabla f\left(x^{k}\right)}
$$

O terceiro método usado foi o Gradiente Generalizado Reduzido (GRG), sendo aplicado em problemas de mistura ternária onde as variáveis independentes implicam numa restrição linear: $\mathrm{x}_{1}+$ $\mathrm{x}_{2}+\mathrm{x}_{3}=1$. A imposição desta restrição reduz $o$ número de graus de liberdade, ou seja, teremos ao invés de três variáveis, duas independentes $\left(\mathrm{x}_{\mathrm{I}}\right)$ e outra dependente $\left(\mathrm{x}_{\mathrm{D}}\right)$ das duas primeiras.

Seja, então, a restrição $\mathrm{h}(\mathrm{x})=\mathrm{x}_{1}+\mathrm{x}_{2}+\mathrm{x}_{3}-1=0$, e a função objetivo $f\left(x_{1}, x_{2}, x_{3}\right)$ :

$$
\mathrm{g}_{\mathrm{r}}^{\mathrm{k}}=\left[\frac{\partial \mathrm{f}(\mathrm{x})}{\partial \mathrm{x}_{\mathrm{I}}}\right]-\left[\frac{\partial \mathrm{h}(\mathrm{x})}{\partial \mathrm{x}_{\mathrm{I}}}\right]^{\mathrm{T}}\left[\left[\frac{\partial \mathrm{h}(\mathrm{x})}{\partial \mathrm{x}_{\mathrm{D}}}\right]^{-1}\right]^{\mathrm{T}}\left[\frac{\partial \mathrm{f}(\mathrm{x})}{\partial \mathrm{x}_{\mathrm{D}}}\right]
$$

onde $g_{r}$ é o gradiente reduzido, que é uma matriz 2x1 relativa às duas variáveis independentes.

Tem-se para as variáveis independentes $\left(\mathrm{x}_{\mathrm{I}}\right)$ :

$$
\mathrm{S}^{\mathrm{k}}=-\mathrm{g}_{\mathrm{r}}^{\mathrm{k}}
$$

Após o cálculo das $\mathrm{x}_{\mathrm{I}}$, a variável $\mathrm{x}_{\mathrm{D}}$ é calculada através de $h(x)$.

\section{Otimização de respostas combinadas}

A idéia básica da função de desejabilidade é transformar um problema de várias respostas em um de uma única resposta por meio de transformações matemáticas. A primeira etapa desta transformação consiste em tornar cada resposta $\mathrm{y}_{\mathrm{m}}(\mathrm{x}), \mathrm{m}=1,2 \ldots, \mathrm{p}$, em funções normalizadas $d_{m}\left[y_{m}(x)\right]$ que possuem valores de imagem entre 0,0 e 1,0.

Como proposto por Derringer e Suich (1980), as funções de normalização ou conveniência são dadas como:

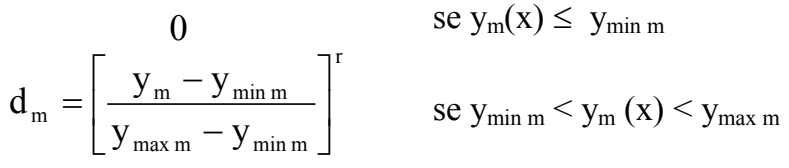

$1 \quad$ se $y_{m}(x) \geq y_{\max m}$

para busca unilateral, e

$$
\begin{aligned}
& d_{m}=\left[\frac{y_{m}-y_{\min m}}{T_{m}-y_{\min m}}\right]^{\mathrm{s}} \quad \text { se } y_{\min m} \leq y_{m}(x) \leq T_{m} \\
& d_{m}=\left[\frac{y_{m}-y_{\max m}}{T_{m}-y_{\max m}}\right]^{t} \quad \text { se } T_{m} \leq y_{m}(x) \leq y_{\max m}
\end{aligned}
$$

$0 \quad$ em outros casos

para busca bilateral.

Onde:

$\mathrm{y}_{\min \mathrm{m}}$ : valor mínimo para a resposta $\mathrm{y}_{\mathrm{m}}$

$\mathrm{y}_{\max m}$ : valor máximo para a resposta $\mathrm{y}_{\mathrm{m}}$

$\mathrm{T}_{\mathrm{m}}$ : valor alvo para a resposta $\mathrm{y}_{\mathrm{m}}$

r,s e t: parâmetros de subjetividade

$\mathrm{m}:$ respostas $(1, \ldots, \mathrm{p})$

Para minimização, na otimização unilateral, os limites superiores e inferiores são invertidos, o que equivale a um complemento $\left(1-\mathrm{d}_{\mathrm{m}}\right)$ para a função de conveniência (SILVA; BORSATO; SILVA, 2000).

Todas as funções de conveniência individuais são combinadas numa função global D ( $\left(\begin{array}{lll}0 & \mathrm{D} & 1\end{array}\right)$, que é definida como a média geométricasdas funções individuais:

$$
D=\left(d_{1} \times d_{2} \times \ldots . . . d_{p}\right)^{1 / p}
$$

Quanto maior o valor de D, mais conveniente é a resposta do sistema, sendo que o valor máximo de $\mathrm{D}$ é a solução otimizada do sistema.

Nas respostas $d_{p}$ com descontinuidades ou singularidades (pontos de inflexão), foi introduzido um polinômio de quarta ordem que se ajusta sobre um pequeno trecho onde está a descontinuidade 
(DEL CASTILLO; MONTGOMERY; MCCARVILLE, 1996):

$$
\mathrm{f}(\mathrm{y})=\mathrm{F}+\mathrm{Gy}+\mathrm{Hy}^{2}+\mathrm{Iy}^{3}+\mathrm{Jy}^{4}
$$

Pode-se observar na Figura 1, que o polinômio entra no trecho entre os pontos a e c, removendo a inflexão que estava presente no ponto b. Para que isso ocorra, porém, as seguintes condições devem ser obedecidas:

$$
\begin{aligned}
& \mathrm{f}(\mathbf{a})=\mathrm{d}(\mathbf{a}) \\
& \mathrm{f}^{\prime}(\mathbf{a})=\mathrm{d}^{\prime}(\mathbf{a}) \\
& \mathrm{f}(\mathbf{c})=\mathrm{d}(\mathbf{c}) \\
& \mathrm{f}^{\prime}(\mathbf{c})=\mathrm{d}^{\prime}(\mathbf{c}) \\
& \mathrm{f}(\mathbf{b})=\mathrm{d}(\mathbf{b})
\end{aligned}
$$

onde: $\mathrm{b}=$ valor objetivo

$\mathrm{a}=\mathrm{b}-\mathrm{b} / 50$

$\mathrm{c}=\mathrm{b}+\mathrm{b} / 50$

sendo a e b denominados como vizinhança do valor objetivo. Tendo um sistema com 5 equações, podemos encontrar 5 incógnitas (F, G, H, I, J) através da manipulação de álgebra linear e assim encontrar o polinômio que satisfaça as restrições acima e remova a descontinuidade da função.

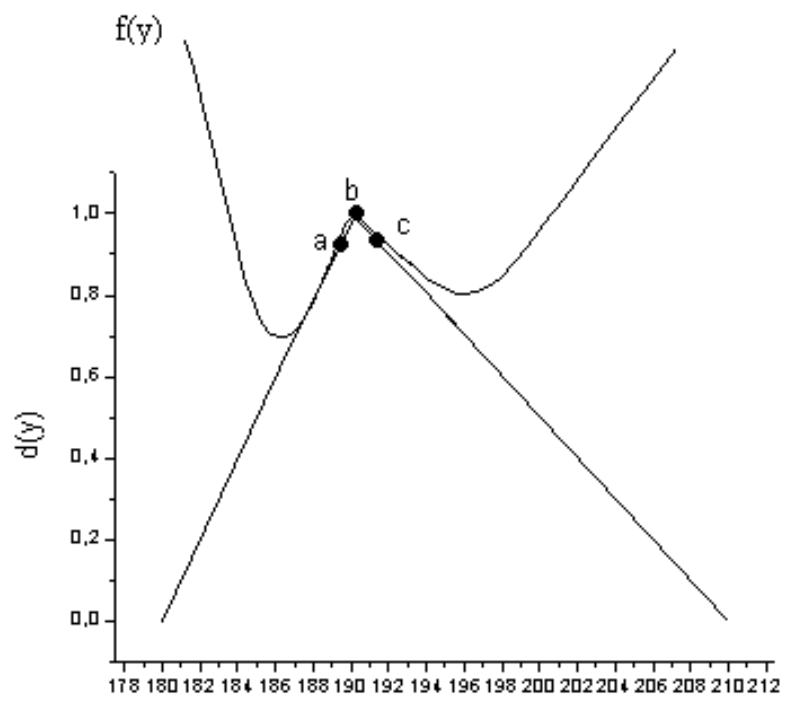

Figura 1. Função de desejabilidade para busca de um valor especifico

A equação contínua de desejabilidade ficará então:

$$
\begin{array}{cl}
\mathrm{d}_{\mathrm{m}}=\left[\frac{\mathrm{y}_{\mathrm{m}}-\mathrm{y}_{\min \mathrm{m}}}{\mathrm{T}_{\mathrm{m}}-\mathrm{y}_{\min \mathrm{m}}}\right]^{\mathrm{s}} & \text { se: } \mathrm{y}_{\min \mathrm{m}} \leq \mathrm{y}_{\mathrm{m}}(\mathrm{x}) \leq \mathbf{a} \\
\mathrm{d}_{\mathrm{m}}=F+G y+H y^{2}+I y^{3}+J y^{4} & \text { se: } \mathbf{a} \leq \mathrm{y}_{\mathrm{m}}(\mathrm{x}) \geq \mathbf{b} \\
\mathrm{d}_{\mathrm{m}}=\left[\frac{\mathrm{y}_{\mathrm{m}}-\mathrm{y}_{\text {max } \mathrm{m}}}{\mathrm{T}_{\mathrm{m}}-\mathrm{y}_{\text {max } \mathrm{m}}}\right]^{\mathrm{t}} & \text { se: } \mathbf{c} \leq \mathrm{y}_{\mathrm{m}}(\mathrm{x}) \leq \mathrm{y}_{\max \mathrm{m}}(\mathrm{x}) \\
0 & \text { outros casos }
\end{array}
$$

\section{Procedimento A - Entrada de dados}

1. Selecionar o tipo de otimização (superfície de respostas ou mistura com três componentes);

2. Fornecer o número de variáveis independentes;

3. Fornecer os limites superior e inferior das variáveis independentes;

4. Definir o número de respostas a serem otimizadas;

5. Definir o objetivo de otimização de cada resposta;

6. Definir os limites superior e inferior e o valor alvo (se for o caso) para cada resposta; 
7. Escolher os parâmetros de subjetividade r,s e t de cada resposta;

8. Entrar com o nome do arquivo para leitura dos coeficientes já salvos ou para salvar a entrada dos novos coeficientes;

9. Entrar com o ponto inicial;

\section{Procedimento B - Otimização método gradiente}

10. Cálculo da desejabilidade do ponto inicial;

11. Cálculo das direções de busca;

12. Cálculo do novo ponto;

13. Cálculo da desejabilidade do novo ponto;

14. Comparação entre a desejabilidade do novo ponto e do ponto anterior, se a desejabilidade do novo ponto for menor, o parâmetro » é reduzido, e o ponto é calculado novamente;

15. Se o novo ponto for melhor que o anterior, e o critério de parada ainda não foi atingido, um novo ponto é calculado voltando ao passo 11 .

16. Na finalização há uma nova comparação entre a desejabilidade de um novo ponto e do ponto imediatamente anterior. Se o critério de convergência é atingido (variação de $10^{-5}$ para $\mathrm{D}$ em relação à dois pontos consecutivos), o ponto ótimo é considerado encontrado.

\section{Resultado e Discussão}

Os sistemas alimentares usados para testar o programa baseado nos métodos de gradiente foram obtidos junto à literatura especializada, apresentam modelagem pela superfície de resposta sendo um deles com delineamento para misturas.

O programa computacional foi desenvolvido em linguagem FORTRAN, e apresenta uma seqüência de entrada de dados que são fornecidos pelo usuário: número de variáveis independentes, limites superior e inferior das variáveis independentes, número de variáveis dependentes, limite superior e inferior das variáveis dependentes que se deseja maximizar, minimizar ou ainda escolher um valor alvo para a otimização.

Para a otimização dos modelos 3.1 e 3.3 , foi utilizada o Gradiente Conjugado em conjunto com o Gradiente tradicional. O Gradiente Conjugado atinge um valor ótimo mais rapidamente, sendo assim, usado primeiro. Porém, o Gradiente tradicional consegue refinar a busca do ótimo devido ao seu $\mathrm{S}^{\mathrm{k}}$ menor e obter respostas melhores que as do Gradiente Conjugado, sendo aplicado após a convergência deste. No modelo 3.2 foi usado o GRG por tratar-se de uma mistura três componentes onde surge uma restrição que está relacionada com a proporção dos ingredientes.

Inativação da lipoxigenase e lípase, preservandose a atividade da fitase durante o processo hidrotérmico da cevada (RUTGERSSON et al., 1997)

A cevada possui altos teores de amido, fibras alimentares, vitaminas e minerais. Entretanto, contém ácido fítico um antinutriente natural presente em grandes quantidades nos cereais. A fitase é uma enzima também de ocorrência natural nos cereais e pode degradar o ácido fítico. Sua ativação, porém, gera também a ativação de lípase, que atua degradando triglicerídios à ácidos graxos, e de lipoxigenase, que atua sobre o substrato da última no processo de oxidação lipídica, diminuindo a vida de prateleira do produto. Esse experimento visou a minimização da atividade de lipoxigenase $\left(\mathrm{y}_{1}\right)$ e lípase $\left(\mathrm{y}_{2}\right)$ e maximização da ativação da fitase $\left(\mathrm{y}_{3}\right)$ em processamento hidrotérmico da cevada.

As variáveis independentes eram adição de ácido lático como acidulante $\left(\mathrm{x}_{1}, \% \mathrm{v} / \mathrm{v},\right)$ temperatura $\left(\mathrm{x}_{2},{ }^{\circ} \mathrm{C}\right)$ e tempo de hidratação $\left(\mathrm{x}_{3}, \mathrm{~min}\right)$.

Os modelos matemáticos com variáveis codificadas, a partir de um delineamento experimental central composto constituído de 17 ensaios sendo 8 fatoriais, 6 axiais e 3 repetições no ponto central são apresentados a seguir: 
$\mathrm{y}_{1}=9,2326-3,8314 \mathrm{x}_{1}-31,2000 \mathrm{x}_{2}-10,4000 \mathrm{x}_{3}-$ $8,7500 x_{1}^{2}+25,0930 x_{2}^{2}+7,0930 x_{3}^{2}+5,75 x_{1} x_{2}-$ $3,0000 \mathrm{x}_{1} \mathrm{x}_{3}+7,2500 \mathrm{x}_{2} \mathrm{x}_{3}$

$\mathrm{y}_{2}=28,8169-12,2000 \mathrm{x}_{1}-27,3000 \mathrm{x}_{2}-7,3000 \mathrm{x}_{3}+$ $4,0704 \mathrm{x}_{1}^{2}+18,5704 \mathrm{x}_{2}^{2}+0,5705 \mathrm{x}_{3}^{2}+9,7500 \mathrm{x}_{1} \mathrm{x}_{2}-$ $2,5000 \mathrm{x}_{1} \mathrm{x}_{3}+5,2500 \mathrm{x}_{2} \mathrm{x}_{3}$

$\mathrm{y}_{3}=52,6046+1,6000 \mathrm{x}_{1}-42,7000 \mathrm{x}_{2}-0,7616 \mathrm{x}_{3}+$ $10,4419 \mathrm{x}_{1}^{2}+27,9419 \mathrm{x}_{2}^{2}-25,7500 \mathrm{x}_{3}^{2}-1,1250 \mathrm{x}_{1} \mathrm{x}_{2}-$ $4,6250 \mathrm{x}_{1} \mathrm{x}_{3}+8,8750 \mathrm{x}_{2} \mathrm{x}_{3}$

Os autores (RUTGERSSON et al. 1997) otimizaram o sistema através da construção de curvas de níveis, para a análise da influência das variáveis tempo e temperatura sobre cada resposta, com a concentração de ácido lático fixada em 1\%.
Posteriormente, as respostas individuais foram combinadas através da superposição dos gráficos, obtendo-se, assim, uma sub-região ótima.

Na otimização conjunta pelo aplicativo OTGRAD, são combinados os métodos de modelagem por superfície de resposta e os métodos de otimização por gradiente, que busca a solução mais desejável para a função de Derringer e Suich (1980).

Os resultados referentes à otimização realizada pelos autores e pelo programa OTGRAD são apresentados na Tabela 1.

A utilização direta do aplicativo tomando o expoente $\mathrm{s}=1$ para todas as respostas, originou valores muito próximos aos obtidos pelos autores, porém o melhor resultado se obteve quando a importância da resposta minimização da atividade da lípase foi destacada. Quando s=3, as condições impostas pelos autores foram facilmente satisfeitas.

Tabela 1. Otimização da concentração de ácido lático $\left(\mathrm{x}_{1}\right)$, temperatura $\left(\mathrm{x}_{2}\right)$ e tempo $\left(\mathrm{x}_{3}\right)$ através do método de gradiente para as respostas lipoxigenase $\left(\mathrm{y}_{1}\right)$, lípase $\left(\mathrm{y}_{2}\right)$ e fitase $\left(\mathrm{y}_{3}\right)$.

\begin{tabular}{c|c|c|c|c|c|c|c}
\hline \multirow{2}{*}{ Método } & $\begin{array}{c}\text { Importância relativa } \\
\text { das respostas } \\
\text { Expoente } \mathrm{s}\end{array}$ & \multicolumn{2}{|c|}{ Variáveis independentes otimizadas } & \multicolumn{3}{|c}{ Respostas ótimas } \\
\cline { 3 - 9 } & $\begin{array}{c}\mathrm{x}_{1} \\
(\% \mathrm{v} / \mathrm{v})\end{array}$ & $\begin{array}{c}\mathrm{x}_{2} \\
\left({ }^{\circ} \mathrm{C}\right)\end{array}$ & $\begin{array}{c}\mathrm{x}_{3} \\
(\mathrm{~min})\end{array}$ & $\mathrm{y}_{1}(\%)$ & $\mathrm{y}_{2}(\%)$ & $\mathrm{y}_{3}(\%)$ \\
\hline Gráfico* & ------------ & 1,00 & $53-58$ & $70-110$ & 0,0 & $<20$ & $>60$ \\
\hline Numérico & $\mathrm{s}=1$ para todas & 1,00 & 54,32 & 87,92 & 0,29 & 22,84 & 72,40 \\
\hline Numérico & $\begin{array}{c}\mathrm{s}=1 \text { para } \mathrm{y}_{1} \mathrm{e} \mathrm{y}_{3} \\
\mathrm{~s}=3 \text { para } \mathrm{y}_{2}\end{array}$ & 1,00 & 55,71 & 92,53 & 0,0 & 18,84 & 63,30 \\
\hline
\end{tabular}

*Fonte: Rutgersson et al. (1997).

Otimização simultânea das respostas na formulação de misturas de proteinas (CASTRO et. al., 2003)

Misturas contendo diferentes proporções de três proteínas [gelatina hidrolisada $\left(\mathrm{HG}, \mathrm{x}_{1}\right)$, glúten de trigo $\left(\mathrm{WG}, \mathrm{x}_{2}\right.$ ) e proteína isolada de soja (SPI, $\left.\mathrm{x}_{3}\right)$ ], foram usadas para preparar uma bebida láctea regularmente consumida em programas institucionais. As proteínas foram misturadas de acordo com o planejamento simplex-centróide (Tabela 2) a fim de explorar os possíveis sinergismos entre os objetivos sensorial, nutricional e econômico. As misturam foram avaliadas sensorialmente (maximização, $\mathrm{y}_{1}$ ), através da determinação da qualidade protéica, utilizando-se ensaios biológicos para obtenção dos índices NPR (razão de eficiência protéica, maximização, $\mathrm{y}_{2}$ ) e PDCAAS (escore químico corrigido pela digestibilidade, maximização, $\mathrm{y}_{3}$ ), e, ainda, pelo custo da formulação (minimização, $y_{4}$ ). 
Tabela 2. Composição das misturas no delineamento experimental

\begin{tabular}{c|c|c|c}
\hline $\mathrm{N}^{\circ}$ do ensaio & $\mathrm{x}_{1}$ & $\mathrm{x}_{2}$ & $\mathrm{x}_{3}$ \\
\hline 1 & 1 & 0 & 0 \\
\hline 2 & 0 & 1 & 0 \\
\hline 3 & 0 & 0 & 1 \\
\hline 4 & $1 / 2$ & $1 / 2$ & 0 \\
\hline 5 & $1 / 2$ & 0 & $1 / 2$ \\
\hline 6 & 0 & $1 / 2$ & $1 / 2$ \\
\hline 7 & $1 / 3$ & $1 / 3$ & $1 / 3$ \\
\hline
\end{tabular}

$* \mathrm{x}_{1}, \mathrm{x}_{2}$ e $\mathrm{x}_{3}$ representam a proporção de derivados de gelatina, glúten e soja respectivamente.

A modelagem matemática com variáveis codificadas $\left(\mathrm{x}_{1}+\mathrm{x}_{2}+\mathrm{x}_{3}=1,0\right)$ resultou:

$\mathrm{y}_{1}=6,08 \mathrm{x}_{1}+2,14 \mathrm{x}_{2}+4,70 \mathrm{x}_{3}-2,08 \mathrm{x}_{1} \mathrm{x}_{2}+2,10 \mathrm{x}_{1} \mathrm{x}_{3}+$ $3,85 \mathrm{x}_{2} \mathrm{x}_{3}$

$\mathrm{y}_{2}=15,62 \mathrm{x}_{1}+22,61 \mathrm{x}_{2}+79,60 \mathrm{x}_{3}+52,72 \mathrm{x}_{1} \mathrm{x}_{2}+$ $43,79 \mathrm{x}_{2} \mathrm{x}_{3}-45,43 \mathrm{x}_{1} \mathrm{x}_{2} \mathrm{x}_{3}$

$\mathrm{y}_{3}=0,61 \mathrm{x}_{1}+1,33 \mathrm{x}_{2}+2,33 \mathrm{x}_{3}+4,63 \mathrm{x}_{1} \mathrm{x}_{2}+1,82 \mathrm{x}_{1} \mathrm{x}_{3}+$ $4,48 \mathrm{x}_{2} \mathrm{x}_{3}$

$\mathrm{y}_{4}=5,75 \mathrm{x}_{1}+4,93 \mathrm{x}_{2}+7,47 \mathrm{x}_{3}$
Os autores otimizaram o sistema através do método de busca direta Simplex com restrição, que também utiliza a função de conveniência de Derringer e Suich (1980). Por isso, foi apresentado o coeficiente de importância relativa das respostas (s). Era de se esperar que o coeficiente de $\mathrm{y}_{1}$ fosse maior que 1 , pois o modelo possui duas respostas para o aspecto nutricional e os valores nutricionais são diretamente dependentes.

$\mathrm{Na}$ otimização pelo programa OTGRAD, foram combinados os métodos de superfície de respostas e o GRG, pois o sistema possui a restrição $\mathrm{x}_{1}+\mathrm{x}_{2}+\mathrm{x}_{3}$ $=1$ já que o modelo é para misturas ternárias.

A otimização pelo OTGRAD satisfez as condições iniciais proposta pelos autores apresentando resultados praticamente idênticos. A otimização desenvolvida pelo OTGRAD e pelos autores citados é mostrada na Tabela 3 .

Tabela 3. Otimização das respostas combinadas na formulação da mistura protéica empregando o método GRG (Gradiente Reduzido Generalizado).

\begin{tabular}{|c|c|c|c|c|c|c|c|c|c|c|c|}
\hline \multirow{2}{*}{ Otimização } & \multicolumn{4}{|c|}{$\begin{array}{l}\text { Importância relativa } \\
\text { (expoente s) }\end{array}$} & \multicolumn{3}{|c|}{ Proporção dos ingredientes } & \multicolumn{4}{|c|}{ Respostas } \\
\hline & $\mathrm{y}_{1}$ & $\mathrm{y}_{2}$ & $y_{3}$ & $\mathrm{y}_{4}$ & $\mathrm{x}_{1}$ & $\mathrm{x}_{2}$ & $\mathrm{x}_{3}$ & $\mathrm{y}_{1}$ & $\mathrm{y}_{2}$ & $\mathrm{y}_{3}$ & $\mathrm{y}_{4}$ \\
\hline Autores* & 2,23 & 1 & 1 & 1 & 0,203 & 0,268 & 0,529 & 4,95 & 59,10 & 2,80 & 6,33 \\
\hline \multirow{4}{*}{ GRG } & 1 & 1 & 1 & 1 & 0 & 0,448 & 0,552 & 4,50 & 64,92 & 2,99 & 6,33 \\
\hline & 1,5 & 1 & 1 & 1 & 0,001 & 0,422 & 0,577 & 4,56 & 66,14 & 3,00 & 6,40 \\
\hline & 2 & 1 & 1 & 1 & 0,144 & 0,313 & 0,543 & 4,82 & 61,27 & 2,88 & 6,43 \\
\hline & 2,23 & 1 & 1 & 1 & 0,203 & 0,267 & 0,530 & 4,95 & 59,12 & 2,79 & 6,45 \\
\hline
\end{tabular}

*Fonte: Castro et. al. (2003). 
Otimização simultânea dos parâmetros de torração de gérmen de milho visando aplicação como ingrediente em alimentos (STRAZZI, 2000)

O gérmen de milho tem sido utilizado, principalmente, para alimentação animal, com baixo valor agregado. Em busca de uma finalidade mais nobre para este produto, o mesmo foi tostado, a fim de proporcionar aroma e sabor de castanha, para ser utilizado como ingrediente em alimentos. Os parâmetros que envolvem o processo de torração foram avaliados considerando, principalmente, as características sensoriais dos produtos torrados. A torração foi realizada em torrador elétrico rotativo. As variáveis independentes estudadas foram temperatura $\left(\mathrm{x}_{1},{ }^{\circ} \mathrm{C}\right)$ e tempo $\left(\mathrm{x}_{2}, \mathrm{~min}\right)$, e as respostas analisadas foram: intensidade de aroma de castanha torrada ( $\mathrm{y}_{1}$, maximização), intensidade de gosto amargo ( $\mathrm{y}_{2}$, minimização) e análise sensorial de cor $\left(\mathrm{y}_{3}\right.$, valor alvo $=$ entre 4 a 6$)$.

Os modelos simplificados com variáveis codificadas (a autora levou em consideração somente os coeficientes significativos em nível de $5 \%, p \leq 0,05$ ) resultaram:

$\mathrm{y}_{1}=5,663-1,317 \mathrm{x}_{1}-0,747 \mathrm{x}_{2}$

$\mathrm{y}_{2}=2,890-1,220 \mathrm{x}_{1}-1,120 \mathrm{x}_{1}^{2}$

$\mathrm{y}_{3}=3,194+1,767 \mathrm{x}_{1}-0,798 \mathrm{x}_{2}$
A autora (STRAZZI, 2000) empregou a metodologia de superfície de respostas (MSR), de acordo com um delineamento $3^{2}$ completo mais duas repetições no ponto central. Através da sobreposição gráfica, foi encontrada uma sub-região que satisfazia todas as condições impostas, e dentro dessa região foi escolhido um ponto que foi definido como ótimo.

A resposta análise sensorial de cor $\left(\mathrm{y}_{3}\right)$ foi baseada em comparações com um padrão de aceitação, sendo que, de acordo com esse padrão, a cor ideal estaria em torno de 5 , porém seria aceitável num intervalo de \pm 1 . Logo, o objetivo da otimização para essa resposta foi buscar o valor de 5. Quando a resposta otimizada é de busca por um valor alvo, a função de Derringer e Suich (1980) se torna descontínua dentro do domínio estudado, sendo necessário a remoção dessa descontinuidade, através da inserção de um polinômio de $4^{\circ}$ grau, para a aplicação do método de gradiente.

A otimização feita pelo OTGRAD obedeceu a todas as condições impostas pela autora, sendo que os resultados experimentais obtidos através da sobreposição gráfica e pela otimização gradiente foram semelhantes em termos das respostas. Apesar de diferir minimamente, o ponto estimado estava dentro da sub-região inicialmente estipulada pela autora como sendo uma região ótima. O parâmetro de importância relativa de todas as respostas foi tomado como 1. O resultado da otimização realizada pela autora e pelo OTGRAD pode ser visto na Tabela 4.

Tabela 4. Otimização dos parâmetros de torração de gérmen de milho para as seguintes análises sensoriais: intensidade de aroma de castanha torrada $\left(\mathrm{y}_{1}\right)$, intensidade de gosto amargo $\left(\mathrm{y}_{2}\right)$ e análise de cor $\left(\mathrm{y}_{3}\right)$.

\begin{tabular}{c|c|c|c|c|c}
\hline \multirow{2}{*}{$\begin{array}{c}\text { Tipo de } \\
\text { otimização }\end{array}$} & \multicolumn{2}{|c|}{ Parâmetros de torração } & \multicolumn{3}{c}{ Respostas ótimas } \\
\cline { 2 - 6 } & $\mathrm{x}_{1}\left({ }^{\circ} \mathrm{C}\right)$ & $\mathrm{x}_{2}(\min )$ & $\mathrm{y}_{1}$ & $\mathrm{y}_{2}$ & $\mathrm{y}_{3}$ \\
\hline Gráfico* & 152 & 29 & 6,60 & 3,17 & 4,27 \\
\hline Gradiente & 152,68 & 30 & 6,76 & 3,29 & 4,47 \\
\hline
\end{tabular}

*Fonte: Strazzi (2000). 


\section{Conclusões}

Geralmente, a otimização multirresposta é complexa e considerada uma atividade de tentativa e erro. A sobreposição gráfica das curvas de nível tem despertado atenção à medida que aumenta o número de respostas, entretanto, se o número for muito grande a superposição gráfica torna-se difícil de realizar. O aplicativo desenvolvido não exclui nem invalida a técnica de superposição gráfica, mas complementa e amplia sua aplicação.

O programa computacional baseado nos métodos de gradiente aplicados à otimização de sistemas alimentares de multirrespostas apresentou resultados esperados em relação a outros métodos de otimização, comprovando sua eficiência e aplicabilidade em sistemas alimentares multirrespostas. Os métodos de gradiente podem também aceitar restrições, o que permite sua aplicação a uma maior variedade de sistemas.

A proposta da inserção de um polinômio de $4^{\circ}$ ordem em um trecho descontínuo da FD mostrou-se válida, tornando possível a aplicação de métodos de gradiente, que fazem o uso da primeira derivada, em equações originalmente descontínuas.

\section{Agradecimentos}

Ao CNPq pela bolsa IC.

\section{Referências}

BARROS NETO, B.; SCARMINIO, I. S.; BRUNS, R. E. Como fazer experimentos: pesquisa e desenvolvimento na ciência e na indústria. Campinas: Unicamp, 2001. 401 p.

BOX, G. E. P.; DRAPER, N. R. Empirical Model-Building and Response Surfaces. New York: John Wiley \& Sons, 1987.

CASTRO, I. A.; SILVA, R. S. F.; TIRAPEGUI, J.; BORSATO, D.; BONA, E. Simultaneous optimization of response variables in protein mixtures formulation: constrained simplex method approach, International Journal of Food Science and Technology, Oxford, v. 38, p. 103-110, 2003.

DEL CASTILlo, E.; MONTGOMERY, D. C.; McCARVILLE, D. R. Modified desirability functions for multiple response optimization, Journal of Quality Technology, Milwaukee, v. 28, n. 3, p. 337-345. 1996.

DERRINGER, G.; SUICH, R. Simultaneous optimization of several response variables. Journal of Quality Technology, Milwaukee, v. 12, n. 4, p. 214-219, 1980.

GUILlOU, A. A.; FLOROS, J. D. Multirresponse optimization minimises salt in natural cucumber fermentation and storage. Journal of Food Science, Chicago, v. 58, p. 1381-1389, 1993.

EDGAR, T. F.; HIMMELBLAU, D. M. Optimization of chemical processes. Singapure: McGraw-Hill, 1989. 652 p.

RUTGERSON, A.; BERGMAN, E. L.; LINGNERT, H.; SANDBERG, A. S. Optimization of temperature, time and latic acid concentration to inactivate lipoxygenase and lipase and preserve phytase activity in barley (cv. Blenhein) during soaking. Cereal Chemistry, Saint Paul, v. 74, n. 6, p. 727-732. 1997.

SILVA, R. A.; BORSATO, D.; SILVA, R. S. S. F. Método simplex supermodificado como estratégia de otimização para respostas combinadas em sistemas alimentares. Ciência e Tecnologia de Alimentos, Campinas, v. 20, n. 3, p. 329-336, 2000.

STRAZZI, S. A. Parâmetros de torração de gérmen de milho visando aplicação como ingrediente em alimentos. 2000. Dissertação (Mestrado)-Universidade Estadual de Londrina, Londrina, 2000. 